\title{
Formación de profesionales para una ciudadanía planetaria. La educación para el desarrollo sostenible en los títulos de grado de la Facultad de Educación de la Universidad Complutense de Madrid
}

\author{
Inmaculada Gómez-Jarabo \\ Universidad Complutense de Madrid/Rector Royo Villanova s/n. Facultad de Educación- \\ Departamento de Estudios Educativos, despacho 4307. Madrid-España. \\ inma.gomez@edu.ucm.es \\ ORCID: https://orcid.org/0000-0003-0159-6910
}

\section{Carmen Saban Vera}

Universidad Complutense de Madrid/Rector Royo Villanova s/n. Facultad de EducaciónDepartamento de Estudios Educativos, despacho 4108. Madrid-España. mcsaban@ucm.es ORCID: https://orcid.org/0000-0002-5356-6126

\section{Bienvenida Sánchez Alba}

Universidad Complutense de Madrid/Rector Royo Villanova s/n. Facultad de EducaciónDepartamento de Estudios Educativos, despacho 4307. Madrid-España.

basalba@edu.ucm.es

ORCID: https://orcid.org/0000-0003-1928-4958

\section{Miguel Barrigüete Garrido}

Universidad Complutense de Madrid/Rector Royo Villanova s/n. Facultad de EducaciónDepartamento de Estudios Educativos, despacho 4107. Madrid-España. miguelbarriguete@edu.ucm.es

ORCID: https://orcid.org/0000-0003-3036-0093

\section{Belén Saénz-Rico de Santiago}

Universidad Complutense de Madrid/ Rector Royo Villanova s/n. Facultad de EducaciónDepartamento de Estudios Educativos, despacho 4108. Madrid-España.

bsaenzri@edu.ucm.es

ORCID: https://orcid.org/0000-0001-9207-889X

[Recibido: 12 Noviembre 2018. Revisado: 6 Febrero 2019. Aceptado: 11Febrero 2019]

Resumen: El presente trabajo de investigación se ha desarrollado en el marco del Proyecto Detección de estándares y criterios de calidad para el desarrollo sostenible y su incorporación a los sistemas internos Garantía de calidad en la Facultad de Educación (Proyectos Innova-Docencia 2017/2018 de la Universidad Complutense de Madrid) cuyo objetivo centra su foco en tratar de dar respuesta a las siguientes cuestiones: ¿son competentes los egresados y egresadas de la Facultad de Educación de la UCM para responder a los diferentes retos, necesidades sociales y ambientales que surgen cada día en nuestro planeta? ¿Desde la propia educación se considera la Educación para el Desarrollo Sostenible como criterio de calidad? 
Desde una metodología cualitativa, se ha realizado un mapeo en base a una selección de fichas docentes y memorias ANECA de las diferentes titulaciones impartidas en la Facultad de Educación: Grado de Maestro Primaria, Grado de Maestro Infantil, Grado de Educación Social y Grado de Pedagogía para identificar las fortalezas y debilidades en la formación de los distintos perfiles profesionales. Para la recogida de datos y posterior análisis se diseñó una matriz tomando como referencia los Objetivos de Desarrollo Sostenible, las competencias desarrolladas por la UNECE y la UNESCO como indicadores de análisis.

Aunque el desarrollo sostenible se muestra presente en las competencias, objetivos, etc. de algunas de las asignaturas analizadas, los resultados apuntan a la necesidad de introducir mejoras relacionadas con la sostenibilidad en las titulaciones impartidas en la Facultad de Educación de la UCM en cumplimiento de los objetivos y metas de la Agenda 2030, para incorporar la dimensión Educación para el Desarrollo Sostenible en cada uno de los perfiles profesionales.

Palabras clave: Educación Desarrollo Sostenible, Objetivos de Desarrollo Sostenible, sostenibilización curricular, ciudadanía planetaria

\section{Formation of professionals for a planetary citizenship. Education for sustainable development} in the graduate degrees of the Faculty of Education of the Complutense University of Madrid

Abstract: This research work has been developed within the framework of the Project Detection of standards and quality criteria for sustainable development and its incorporation into internal systems Quality assurance in the Faculty of Education (Innova-Docencia Projects 2017/2018 de the Complutense University of Madrid) whose objective focuses on trying to answer the following questions: are graduates and graduates of the Faculty of Education of the UCM competent to respond to the different challenges, social and environmental needs that arise each day on our planet? Is education for sustainable development considered as a quality criterion?

From a qualitative methodology, a mapping has been constructed based on a selection of educational records and ANECA memories of the different degrees taught in the Faculty of Education: Degree of Primary Teacher, Degree of Infant Teacher, Degree in Social Education and Degree in Pedagogy to identify the strengths and weaknesses in the formation of the different professional profiles. For the collection of data and subsequent analysis, a matrix was designed taking as reference the Sustainable Development Goals, the competences developed by UNECE and UNESCO as indicators of analysis.

Although sustainable development is present in the competences, objectives, etc. of some of the subjects analyzed, the results point to the need to introduce improvements related to sustainability in the degrees taught at the Faculty of Education of the UCM in compliance with the objectives and goals of the 2030 Agenda, to incorporate the Education dimension for Sustainable Development in each of the professional profiles.

Keywords: Sustainable Development Education, Sustainable Development Goals, curricular sustainability, planetary citizenship

Para citar este artículo: Gómez-Jarabo, I., Saban Vera, C., Sánchez Alba, B., Barrigüete Garrido, M. y SaenzRico de Santiago, B. (2019). Formación de profesionales para una ciudadanía planetaria. La educación para el desarrollo sostenible en los títulos de grado de la Facultad de Educación de la Universidad Complutense de Madrid. Revista de Educación Ambiental y Sostenibilidad 1(1), 1205. doi: 10.25267/Rev_educ_ambient_sostenibilidad.2019.v1.i1.1205

\section{Introducción}

Han sido muchos los esfuerzos internacionales a favor de la sostenibilidad desde que la Comisión Bruntland introdujera el concepto de Desarrollo Sostenible y las Naciones Unidas proclamaran los Objetivos de Desarrollo del Milenio y la Década de la Educación para el Desarrollo Sostenible. En 2015 se aprobaron los 17 Objetivos de Desarrollo Sostenible (ODS) y 169 metas, desarrolladas en La declaración de Naciones Unidas Transformando nuestro mundo: la Agenda 2030 para el Desarrollo Sostenible a partir de la Agenda 2030. 
Los ODS reconocen la importancia de la educación para el desarrollo sostenible a través del ODS 4, que exige proporcionar educación de calidad inclusiva e igualitaria y promover oportunidades de aprendizaje permanente para todos. Algunas de las metas dentro de este objetivo requieren la acción explícita de las universidades, y otras tienen relevancia directa para las actividades de aprendizaje y enseñanza dentro de las aulas universitarias.

En el ámbito universitario, dicha Agenda sostiene que se deberá procurar el acceso para todos y todas a una formación técnica, profesional y superior de calidad (ONU, 2015). Para lograrlo, las titulaciones deberán incorporar competencias en sostenibilidad que fortalezcan los perfiles profesionales y que sirvan para que los futuros profesionales sean capaces de responder a los retos que plantea una sociedad en constante cambio y evolución. En este sentido, la educación superior juega un papel crucial en la formación de profesionales que deberán adquirir competencias y conocimiento para actuar ante situaciones cada vez más complejas, transdisciplinares y transnacionales. Reconocer esta situación implica que las instituciones de Educación Superior tienen que transformar el modo en que el propio conocimiento se genera y comparte. "Es necesario desarrollar competencias que permitan a las generaciones actuales comprender, empatizar y practicar valores colectivos así como principios que puedan liderar acciones encaminadas a una mejor calidad de vida, respetando las limitaciones naturales del planeta" (UNU-IAS, 2014, p. 8).

Organismos internacionales como Sustainable Development Solutions Network (SDSN), Future Earth, la Organización de las Naciones Unidas para la Educación la Ciencia y la Cultura (UNESCO) y organismos nacionales como CRUE, CADEP (en la actualidad CRUE-Sostenibilidad) y el OCUD apuntan a que los grados universitarios deben repensar sus diseños curriculares, incorporando competencias genéricas vinculadas a la transmisión de valores para formar ciudadanos responsables y comprometidos con el desarrollo sostenible para la transformación desde la acción.

Existe un número creciente de estudios que abordan cómo integrar la sostenibilidad en la educación superior, y cómo se debería concretar en los diferentes marcos curriculares. En este sentido destacamos aportaciones como las de Sterling (2010. Cit. en UNU-IAS, 2014, p. 64) que nos señala que se trata de buscar un equilibrio entre la dominancia del antiguo paradigma y la transformación hacia un paradigma holístico, sistémico y participativo (Tabla 1).

Tabla 1. Integración de la sostenibilidad en la educación superior transición al cambio.

\begin{tabular}{ll}
\hline DESDE... & HACIA... \\
\hline Aprendizaje transmisivo & Aprendizaje por descubrimiento \\
\hline Aprendizaje centrado en el enfoque del docente & Centrado en el discente \\
\hline Aprendizaje individual & Aprendizaje colaborativo \\
\hline Aprendizaje básicamente teórico & $\begin{array}{l}\text { Aprendizaje orientado a través de la práctica y la } \\
\text { experiencia }\end{array}$ \\
\hline Centralizado en la acumulación de conocimientos & Centrado en un aprendizaje auto-regulado \\
\hline Énfasis de los objetivos cognitivos & Objetivos cognitivos, actitudinales \\
\hline Enseñanza/aprendizaje institucionalizado & Inclusión de aprendizajes y experiencias no formales \\
\hline Bajo nivel de aprendizaje cognitivo & Mayor nivel de aprendizaje cognitivo \\
\hline
\end{tabular}


Desde ese punto de partida, el presente trabajo aborda el análisis de una selección de fichas docentes de las titulaciones de Maestro en Educación Infantil, Maestro en Educación Primaria, Pedagogía y Educación Social, todas ellas impartidas en la Facultad de Educación de la Universidad Complutense de Madrid.

La educación, la investigación, la innovación y el liderazgo serán esenciales para los nuevos desafíos del planeta. Las universidades, debido a su labor de generación, difusión y transferencia del conocimiento están llamadas a convertirse en agentes de cambio y transformación. Desde su Responsabilidad Social debe proporcionar respuestas a los problemas de la sociedad, experimentar científica y tecnológicamente las soluciones a dichos problemas y capacitar al capital humano que debe emprender el cambio, convirtiéndose en un agente de cambio generacional.

\section{Diseño de la investigación}

Para la consecución de los objetivos pretendidos, se establecieron una serie de criterios de análisis a partir del concepto de sostenibilidad, las competencias UNECE (2013), UNESCO (2014), y los ODS (ONU, 2015), el modelo Universidad ONU y las directrices marcadas en esta materia por la SDSN (Figura 1).

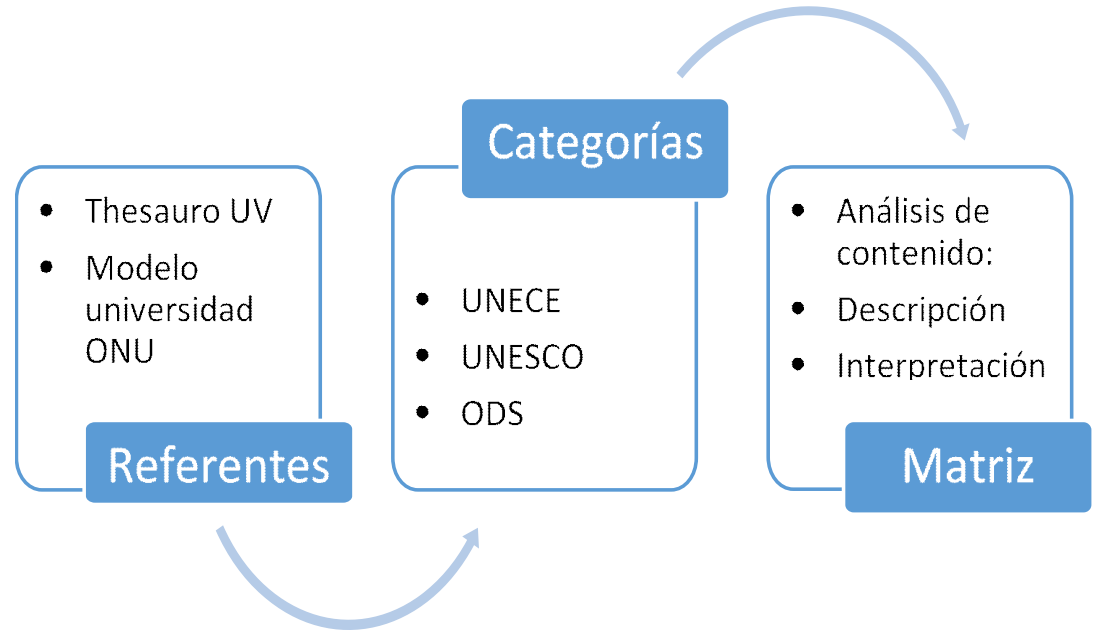

Figura 1. Referentes para la elaboración de la matriz análisis

Se configuró una matriz en la que registrar la información procedente del análisis documental y facilitar de esta forma el proceso de mapeo de las Titulaciones desde un enfoque cualitativo, descriptivo en la cual quedan recogidos todos los criterios de análisis (figura 2).

La muestra objeto de análisis está conformada por las 5 memorias ANECA correspondientes a cada una de las Titulaciones que se imparten en la Facultad de Educación de la Universidad Complutense de Madrid (Grado Maestro Educación Primaria, Grado Maestro Educación Infantil, Grado de Pedagogía y Grado en Educación Social) y por una total de 12 asignaturas representativas de las asignaturas de carácter básico, obligatorias y optativas. Las técnicas de recogida de datos que se han utilizado en el presente proyecto son de carácter mixto y se basó en la complementariedad de técnicas diferentes. Para el tratamiento cuantitativo de datos se trabajó con la herramienta SPSS, mientras que, para el análisis de contenido, se 
utilizó el programa Atlas.Ti. A continuación, en la figura 3, se presenta un esquema resumen del proceso de la investigación realizada.

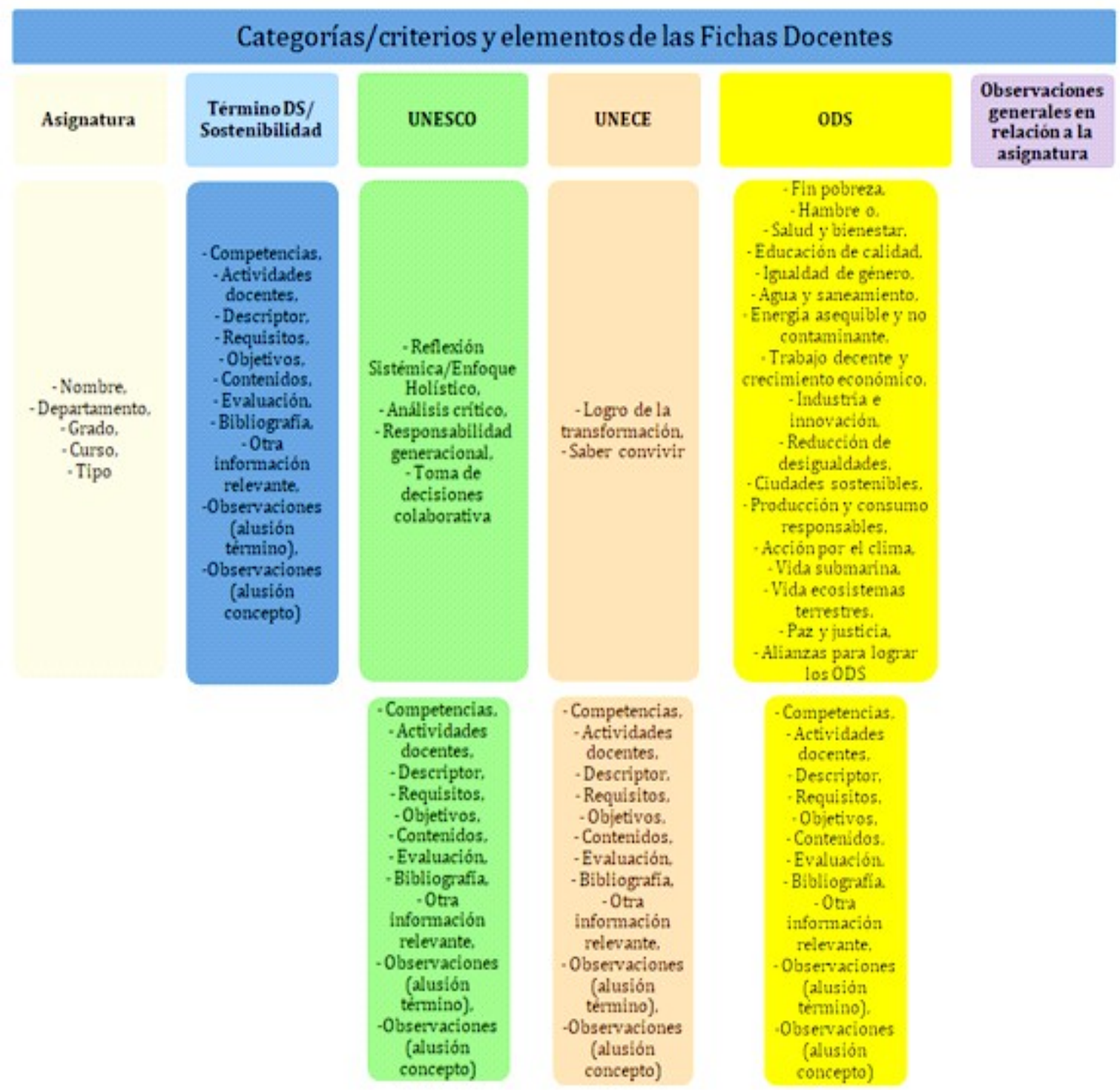

Figura 2. Matriz por categorías/criterios y elementos de las Fichas Docentes

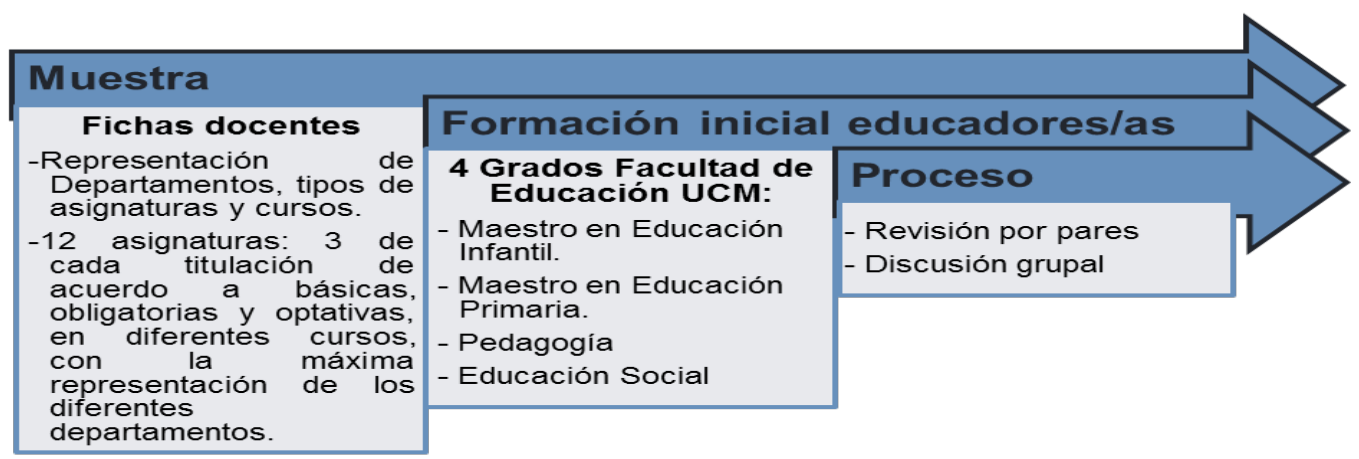

Figura 3. Resumen del proceso de la investigación 


\section{Análisis y Resultados}

Del estudio realizado, se destaca una carencia de abordaje de forma holística en la formación que vaya más allá de la inclusión de competencias genéricas y/o transversales en las fichas docentes de las asignaturas analizadas, lo que muestra la necesidad de revisar y actualizar el perfil de los futuros egresados y por lo tanto profesionales de la Educación en todos sus apartados, para que éstas sean más coherentes con los principios que inspiran la sostenibilidad, con los ODS y con las metas de la Agenda 2030.

Del primer análisis de carácter cuantitativo, cabe destacar un resultado aparentemente positivo. De hecho, si tomamos como referencia los criterios analizados en las asignaturas analizadas (Ver Figura 4) aparecen explícitos tanto el término desarrollo sostenible, como las competencias señaladas por la UNESCO, la UNECE así como una presencia (algo menor) de los propios ODS.

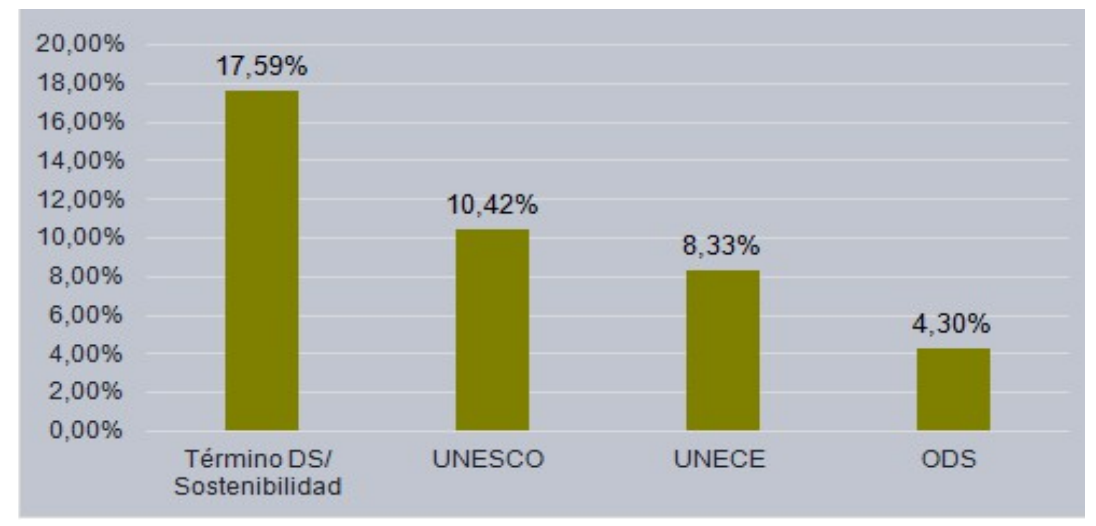

Figura 4. Criterios presentes en las asignaturas analizadas

Decimos aparente, porque ello no conlleva en los contenidos de las asignaturas propuestas para su análisis a través de las fichas o guías docentes, un tratamiento en profundidad. Si tomamos como valor absoluto la presencia del término desarrollo sostenible o sostenibilidad $(17,59 \%)$ podemos concluir que existe una preocupación por su introducción en las fichas docentes del enfoque Educación para el Desarrollo Sostenible. Ahora bien, si vamos avanzando en el análisis, vemos cómo dicha presencia va disminuyendo de manera paulatina en el modo de cómo se concreta el término en las asignaturas, hasta llegar al análisis de los propios ODS, en donde queda reducida a un $4,30 \%$ en su globalidad. En posteriores figuras, podemos ver cómo se distribuye la presencia y/o ausencia de todos los criterios sometidos a análisis.

En la figura 5, se pone en relación la presencia del concepto de sostenibilidad, las competencias UNECE (2013), UNESCO (2014), y los ODS (ONU, 2015), en las 12 asignaturas seleccionadas. En la misma, podemos advertir diferentes niveles de presencia que van desde la inexistencia en las materias de alguno de los ODS, como en el ODS 2 (Hambre 0), y el ODS 6 (Agua y saneamiento) hasta la mayor presencia en éstas del término DS, participando en la asignatura de Desarrollo profesional de docentes y educadores, con más de un $50 \%$. En cuanto las competencias, la más referida en todas las materias es la competencia de análisis crítico, seguida de saber convivir en 9 de las 12 materias analizadas, con un porcentaje superior al $10 \%$. En cuanto a la presencia de los ODS el de mayor referencia se observa que es el $n=4$, Educación de calidad, en 8 de las 12 materias y con una media de presencia en torno al 
12\%, seguida del no 10, Reducción de las desigualdades referido en 6 materias y con un porcentaje entorno al 11\%. En cuanto a la aparición media de los ODS, sí podemos advertir, excepto el $n^{\circ} 2$ como ya se señaló, que en medida desigual están presente en el conjunto de todas las disciplinas, aunque de formar muy tímida con una media aproximada al $5 \%$.

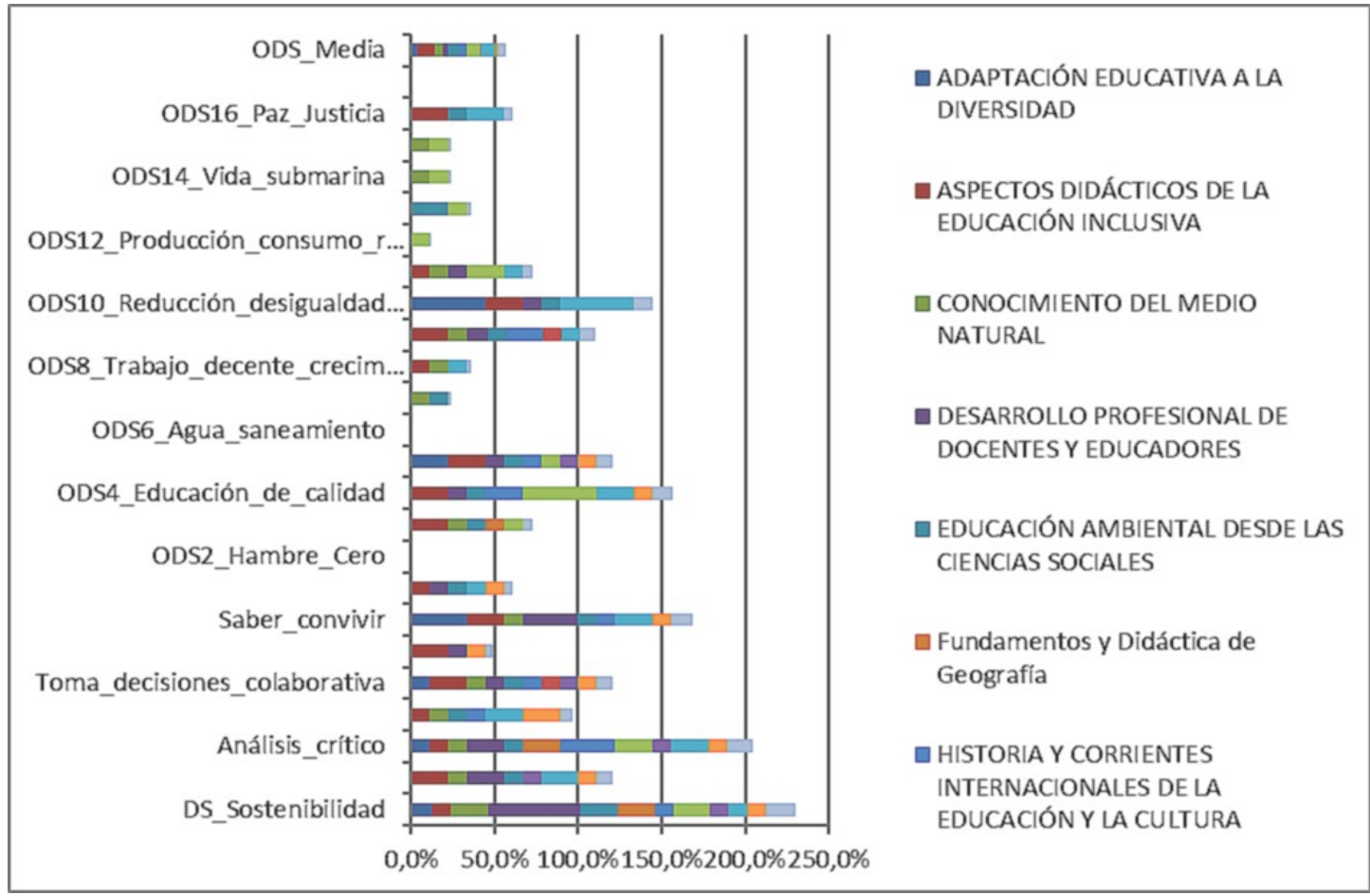

Figura 5. Presencia de criterios por asignatura.

Los datos obtenidos respecto a la relación y presencia de criterios y elementos analizados en las fichas docentes (figura 6) muestran un resultado sumamente desigual. Por un lado, donde más aparecen las competencias UNECE Y UNESCO, los ODS y el propio término de sostenibilidad es en las competencias de las fichas docentes. No obstante, la mayor presencia se encuentra en el término de sostenibilidad (91.7\%), en la competencia de análisis crítico (91.7\%), toma de decisiones colaborativa (66.7\%), saber convivir (66.7\%) y reflexión sistémica (58.3\%) y en el ODS 5 -igualdad de género con un 66.7\%. Es destacable que, por el contrario, muchos de los ODS (2 -hambre 0-, 6 -agua limpia y saneamiento-, 7 -energía asequible y no contaminante-, 12 -producción y consumo responsables-, 13 -acción por el clima-, 14 -vida submarina-, 15 -vida de ecosistemas terrestres- y 17 -alianzas para lograr los objetivos-) no se encuentran presentes en las competencias de las fichas docentes analizadas, lo que hace que la media de aparición de los ODS en las competencias baje a un 15.9\%. En relación a los ODS, también es preciso destacar que un $17.64 \%$ de ellos no se encuentran presentes en ninguno de los elementos analizados de las fichas docentes. Es el caso de los ODS 2 (hambre 0), 6 (agua limpia y saneamiento) y 17 (alianzas para lograr los objetivos). Por tanto, nos encontramos ante un claro predominio de la dimensión de justicia social frente a la de biodiversidad, y economía sostenible. Por otro lado, en el resto de apartados de las fichas docentes se observa una tímida presencia de los criterios y elementos analizados, cuyo porcentaje más alto de aparición asciende a un 41.7\% (presencia del ODS 4 -educación de calidad- en los objetivos de las fichas docentes), siendo por 
mucha diferencia un porcentaje alto de aparición frente al resto de los observados, que se encuentran entre el $0 \%$ y el $17.8 \%$.

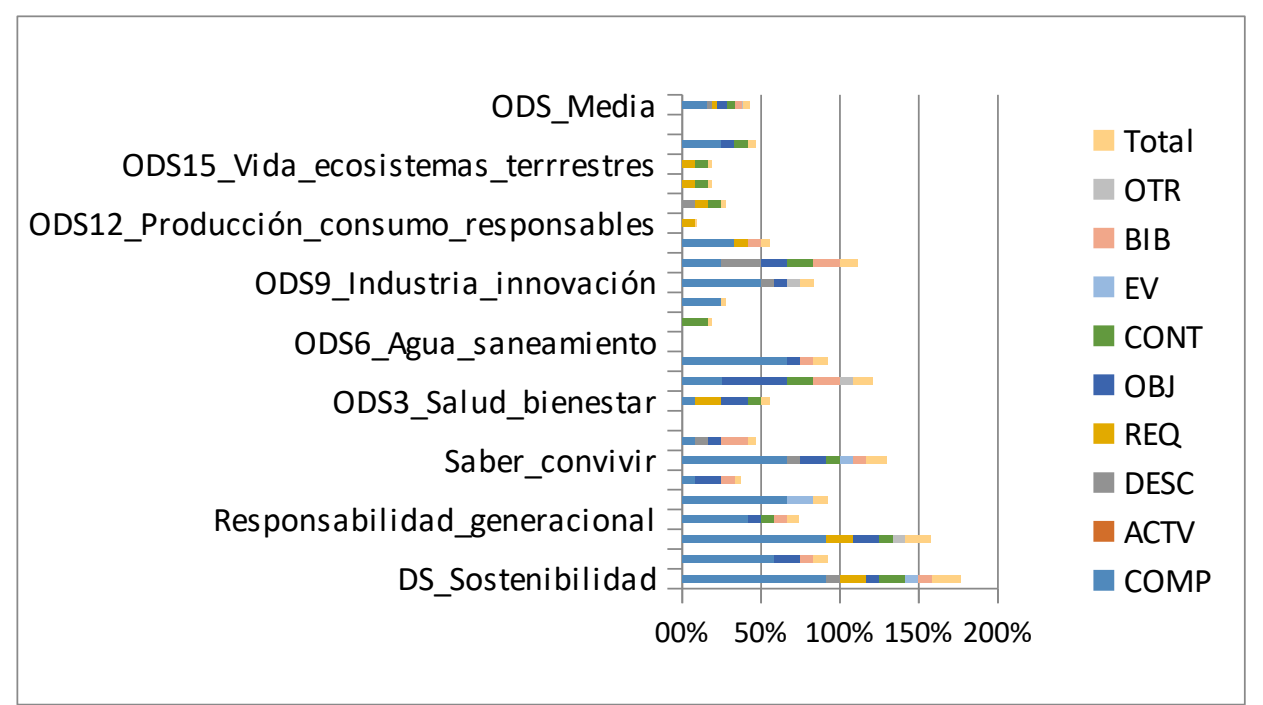

Figura 6. Relación y presencia de criterios análisis en las fichas docentes.

Tomando como referencia las directrices marcadas por la UNESCO para la Educación para el Desarrollo sostenible, la meta 8 marca la importancia de las competencias en el marco de la formación inicial y aprendizaje a lo largo de la vida, tal y como se recoge a continuación (UNESCO, 2014, p. 9):

Lograr que todos los jóvenes (de entre 15 y 24 años de edad) y adultos tengan posibilidades de adquirir con el apoyo de entornos de aprendizaje seguros e inclusivos que tengan en cuenta las cuestiones de género, conocimientos y competencias pertinentes que contribuyan a su realización personal, la paz y un mundo equitativo y sostenible.

Esta tercera parte del estudio realizado, centró su foco de estudio en la revisión de las competencias de cada una de las titulaciones a partir del análisis de contenido de las fichas docentes y de los perfiles profesionales para cada Titulación de las memorias ANECA, en relación a las competencias que actualmente definen cada uno de los perfiles de los futuros profesionales de la educación tanto en el ámbito formal como en el no formal.

En las memorias de cada una de las Titulaciones se concluye en una presencia desigual y sesgada del objeto de estudio. A continuación, destacamos aquellas que aparecen con una alta presencia en las fichas analizadas. Así, resaltamos:

- Competencias Generales:

- CG11.Mediar en situaciones de riesgo y conflicto.

- CG 8. Manejar las herramientas adecuadas para la identificación de problemas sociales y la investigación sobre ellos (...) para emitir juicios argumentados que permitan mejorar los sistemas sociales y la práctica educativa.

- Competencias Transversales:

- CT16 Fomentar la educación democrática de la ciudadanía y la práctica del pensamiento social crítico. 
- CT15 Reconocer la mutua influencia entre ciencia, sociedad y desarrollo tecnológico (...) para procurar un futuro sostenible.

- CT3. Analizar de forma reflexiva y crítica las cuestiones más relevantes de la sociedad actual (...): relaciones de género e intergeneracionales, multiculturalidad e interculturalidad, discriminación e inclusión social y desarrollo sostenible.

- Competencias de materia y/o asignatura:

- CM6.1.1 Analizar de forma reflexiva y crítica las cuestiones más relevantes de la sociedad actual que afectan a la educación familiar y escolar: impacto social y educativo de los lenguajes audiovisuales y de las pantallas; cambios en las relaciones de género e intergeneracionales, multiculturalidad e interculturalidad, discriminación e inclusión social y desarrollo sostenible.

- CM13.2.1. Elaborar propuestas didácticas en relación con la interacción ciencia, técnica, sociedad y desarrollo sostenible.

Podemos afirmar que sobre el papel podríamos considerar que tras el análisis realizado, y en base a las competencias seleccionadas y descritas anteriormente se podría llegar a la conclusión de que en la Facultad de Educación de la Universidad Complutense de Madrid, se realiza una formación basada en una Educación para el Desarrollo Sostenible, pues vemos en este grupo de competencias tanto conceptos, habilidades y actitudes representativas del eje biodiversidad y del eje justicia social, que caracterizan junto con la economía sostenible el concepto de sostenibilidad. ¿Por qué no? ¿Por qué se sugiere una re-conceptualización de las guías docentes actuales?, ¿Quizás no es suficiente al carecer de un elemento que posibilite la construcción holística del aprendizaje desde la sostenibilidad?

El grupo de expertos de la UNECE en competencias educativas para el Desarrollo Sostenible establece como competencias clave para la Educación, UNECE (2012), para incorporar el paradigma del Desarrollo Sostenible la necesidad de ofertar una educación con un enfoque holístico, que busque el abordaje del pensamiento y la práctica de una manera integrada; una educación que capacite para visualizar el cambio: un profesional que explore futuros alternativos, aprende del pasado e inspira compromiso con el presente. Con un propósito final que es de lograr la transformación, lo cual implica cambiar la forma en que las personas aprenden e innovar en las metodologías y técnicas que apoyan el proceso de enseñanza y aprendizaje.

Como competencias clave o básicas que todo sistema educativo debe hacer propias y que posibilitaran la adquisición de una amplia gama de experiencias de aprendizaje la UNECE (2012, p. 13) establece las siguientes categorías:

A.- Aprender a saber se refiere a comprender los desafíos que enfrenta la sociedad tanto a nivel local como globalmente y el papel potencial de educadores y aprendices.

B.- Aprender a hacer se refiere al desarrollo de habilidades prácticas y la capacidad de acción en relación con educación para el desarrollo sostenible. 
C.- Aprender a vivir juntos contribuye al desarrollo de asociaciones y una apreciación de interdependencia, pluralismo, entendimiento mutuo y paz.

D.- Aprender a ser aborda el desarrollo de los atributos personales y la capacidad de actuar con mayor autonomía, juicio y responsabilidad personal en relación con el desarrollo sostenible.

A nuestro entender y desde la mirada de capacitar para visualizar el cambio que proclama la UNESCO, consideramos que de ahora en adelante los planes de estudio deberán plantearse un cambio profundo en el peso de las competencias en los perfiles formativos, asumiendo que tanto el contenido conceptual como procedimental específico de una materia o asignatura debe desarrollarse en las competencias denominadas específicas; dando prioridad a la incorporación en las competencias genéricas o básicas el tipo habilidades cognitivas y valores que requiere el nuevo escenario marcado por un mundo en crisis, dentro de la denominada revolución tecnológica que requiere de profesionales sostenibles tanto en su ejercicio profesional como en su desarrollo activo como ciudadanos; no olvidando como referente la puesta en juego de las habilidades cognitivas y meta cognitivas necesarias para la construcción holística del pensamiento, la capacidad crítica y el pensamiento creativo necesario para conseguir la transformación personal y social.

\section{Discusión/Conclusiones}

En el año 2012 la Comisión Sectorial de Calidad Ambiental, Desarrollo Sostenible y Prevención de Riesgos (CADEP) aprobó una serie de directrices para incluir la sostenibilidad en todos los planes de estudio de titulaciones y asignaturas etc. para impulsar un cambio cultural hacia la sostenibilidad. Así mismo, en 2016 "el Observatorio de Cooperación Universitaria al Desarrollo (OCUD) y la CRUE ampliaban y ratificaban dichas directrices para integrar la Agenda 2030 en la docencia universitaria" (Sánchez, Gómez-Jarabo, Saban y Sáenz-Rico, 2017, p. 113). Por tanto, el cambio de paradigma que supone la introducción de los ODS y la Agenda 2030 en la Educación Superior, requiere de una revisión en profundidad de las Memorias Verifica de los títulos de grado impartidos en la Facultad de Educación de la UCM. De ello se desprende la necesidad de actualizar y reformular explícitamente los planes universitarios para incluir las competencias en sostenibilidad. A partir de la revisión documental realizada, cabe destacar que en la mayoría de las titulaciones analizadas, la competencia de mayor concurrencia hace referencia al análisis crítico, pero en detrimento de otras, fundamentales para la acción y la transformación, como son aquellas de carácter actitudinal e incluso, procedimental, encaminadas al logro de la transformación social y de una antropo-ética en el ejercicio profesional mediante un Desarrollo Sostenible. Asimismo, se evidencia una carencia de abordaje de forma holística que vaya más allá de la inclusión de competencias genéricas y/o transversales, lo que muestra la necesidad de revisar y actualizar, a ser posible desde una perspectiva interdisciplinar y/o transdiciplinar, las fichas docentes de las distintas asignaturas en todos sus componentes, para que éstas sean más coherentes con los principios que inspiran la sostenibilidad.

Los resultados obtenidos en el proyecto que dio origen a este trabajo concuerdan con los de otras investigaciones, al reflejar la tendencia de diferentes administraciones 
para implementar la sostenibilidad en el desarrollo curricular de los estudios universitarios, y percibir a la universidad como un agente de cambio (Sánchez et al. 2017). En este mismo sentido se expresan algunas metas del ODS 4 (Educación de Calidad) como son, por ejemplo, la referida a garantizar de aquí a 2030, el acceso igualitario de todos los hombres y las mujeres a una formación técnica, profesional y superior de calidad, incluida la enseñanza universitaria (meta 4.3), o la meta 4.5 que indica que "de aquí a 2030, se deben eliminar las disparidades de género en la educación y asegurar el acceso igualitario a todos los niveles de la enseñanza y la formación profesional para las personas vulnerables, incluidas las personas con discapacidad, los pueblos indígenas y los niños en situaciones de vulnerabilidad" (ONU, 2015, p. 19).

Las asignaturas que configuran los planes de estudio se prestan a un abordaje tanto del DS como de los ODS mucho más amplio que el actual, que permitieran incorporar al perfil profesional del futuro egresado competencias para dar respuesta a las necesidades tanto locales como planetarias que en los próximos años marcarán un nuevo escenario, en el que será necesario asegurar que todos los alumnos adquieran los conocimientos teóricos y prácticos necesarios para promover el desarrollo sostenible, entre otras cosas mediante la educación para el desarrollo sostenible y los estilos de vida sostenibles, los derechos humanos, la igualdad de género, la promoción de una cultura de paz y no violencia, la ciudadanía mundial y la valoración de la diversidad cultural y la contribución de la cultura al desarrollo sostenible. En este sentido, como apunta la meta 4.7 (ONU, 2015) y los estudios sobre ciudadanía mundial que cada vez destacan más la importancia de incluir, más allá de la idea de estado-nación, un sentido de pertenencia a una comunidad más amplia y a una humanidad común la educación a lo largo de toda la vida se configura como el marco para lograr las siguientes metas:

- Comprender las estructuras de gobernanza mundial, los derechos y las responsabilidades internacionales, los problemas mundiales y las relaciones entre los sistemas y procesos mundiales, nacionales y locales;

- Reconocer y apreciar la diferencia y las identidades múltiples, por ejemplo en materia de cultura, lengua, religión y género y nuestra humanidad común, y adquirir aptitudes para vivir en un mundo cada vez más diverso;

- Adquirir y aplicar competencias críticas para el conocimiento cívico, por ejemplo, indagación crítica, tecnología de la información, competencias básicas en medios de comunicación, pensamiento crítico, adopción de decisiones, solución de problemas, negociación, consolidación de la paz y responsabilidad personal y social;

- Reconocer y examinar creencias y valores y la manera en que las percepciones acerca de la justicia social y el compromiso cívico influyen en la adopción de decisiones políticas y sociales;

- Desarrollar actitudes de interés y empatía respecto al prójimo y el medio ambiente, y de respeto por la diversidad;

- Adquirir valores de equidad y justicia social, y capacidades para analizar críticamente las desigualdades basadas en el género, la condición socioeconómica, la cultura, la religión, la edad y otros factores; 
- Interesarse en las cuestiones mundiales contemporáneas en los planos local, nacional y mundial, y aportar contribuciones propias de ciudadanos informados, comprometidos, responsables y reactivos (UNESCO, 2015, p. 16).

La importancia del vínculo entre ODS y educación a lo largo de toda la vida se ha visto reforzado recientemente a través del reconocimiento del aprendizaje a lo largo de toda la vida como motor de la sostenibilidad medioambiental, social, cultural y económica (Ver Declaración de Cork, 2017: Ciudades del Aprendizaje. Objetivos globales, acciones locales: hacia un aprendizaje a lo largo de toda la vida para todos en 2030. En UIL-UNESCO, 2017).

Sin embargo, en realidad se abordan de manera parcial, e incluso sesgada en los planteamientos actuales de la formación inicial que se imparte en la Universidad. Constituiría un contexto idóneo para actualizar los objetivos y especialmente los contenidos tanto conceptuales como procedimentales de la asignatura desde el marco de los ODS y servir como marco de referencia tanto para la formación inicial superior como para el fortalecimiento de los perfiles profesionales de las titulaciones. Por ello, se propone la necesidad de actualizar las fichas docentes en todos sus apartados no solo a nivel competencial sino también, y especialmente, en relación a otros elementos fundamentales como son la evaluación y las metodologías aplicadas como paso fundamental a la hora de plantear acciones para la mejora de la calidad y la transformación hacia unas enseñanzas desde el marco de la sostenibilidad.

De acuerdo con otros planteamientos internacionales como el modelo de universidad de Naciones Unidas (UNU-IAS, 2014) o el propuesto por la Sustainable Development Solutions Network (SDSN, 2017), y teniendo en cuenta lo que plantean expertos en términos como los que a continuación se recogen:

Superadas en lo fundamental las tesis que han venido negando la existencia de la crisis ecológica y su relación con la actividad humana y más allá de las profundas y complejas contradicciones que atraviesan el mundo, adquiere ahora importancia central el debate sobre cuestiones tan trascendentales como: la capacidad/ incapacidad de la tecnociencia para resolver, por sí sola, las crecientes contradicciones entre los patrones de desarrollo vigentes y la preservación de los sistemas de vida; la posibilidad/imposibilidad de que el proceso de cambio sea liderado por los mismos conglomerados de poder político y económico que nos han traído hasta aquí; la confianza en que las transformaciones precisas puedan ser impulsadas por un capitalismo (de mercado o de Estado) globalizado y alimentado por lógicas de acumulación y consumo ilimitados/indiscriminados o la necesidad de alumbrar nuevos paradigmas, principios y lógicas de actuación que permitan vivir dignamente sin dañar la biosfera; o, incluso, si las "ventanas de oportunidad" para el cambio siguen abiertas o, por el contrario, pudieran haberse cerrado convirtiendo en poco verosímiles los intentos de reconducir ordenadamente los procesos hacia el colapso ecosocial. (Prats, Herrero y Torrego, 2017, pp. 5-6).

Se debería, pues, potenciar claramente el pensamiento y la reflexión crítica sobre las desigualdades y el desarrollo bajo el paradigma de una enseñanza integral, humanista 
y a lo largo de toda la vida. Dicho enfoque integral deberá combinar estrategias como: enseñanza aprendizaje, investigación, gobernanza y liderazgo social.

Las mejoras, si en realidad pretendemos la efectividad de los profesionales de la educación en la transformación del Mundo desde una perspectiva sostenible que los habilite para la acción y de ella surja dicha transformación, no solo deben reflejarse en elementos de las fichas y guías docentes, sino también y claramente en la incorporación de los ODS como tales. Para la consecución de este abordaje holístico hay que profundizar claramente en habilidades y competencias clave, relevantes para abordar todos los ODS desde procesos de pensamiento sistémico, crítico, autoconciencia, resolución integral de problemas y capacidad de anticipación; competencias normativas, estratégicas y de colaboración; creatividad, espíritu emprendedor, y habilidades de aprendizaje, responsabilidad social, capacidad para generar sinergias y sentirse cómodo en entornos interdisciplinarios para dar respuesta, no sólo en el plano simbólico sino también en el material, al logro de las metas 2030.

\section{Referencias bibliográficas}

ONU (2015). Objetivos de Desarrollo Sostenible. 17 Objetivos para cambiar el mundo. Recuperado de http://www.un.org/sustainabledevelopment/es/2015/09/laasamblea-general-adopta-la-agenda-2030-para-el-desarrollo-sostenible/

Prats, F. Herrero, Y. y Torrego, A. (coords.) (2017). La Gran Encrucijada. Sobre la Crisis Ecosocial y el Cambio de Ciclo Histórico. Madrid: Edit. Libros en Acción.

Sánchez; B., Gómez-Jarabo, I., Saban, C. y Sáenz-Rico, B. (2017). Sostenibilización del perfil profesional del educador social. Necesidades y demandas compartidas. Revista Iberoamericana de Educación, (73), 109-130.

Sterling, S. (2010). Transformative Learning and Sustainability: sketching the conceptual ground. Learning and Teaching in Higher Education, (5), 17-33.

SDSN (2017). Cómo empezar con los ODS en las Universidades. Una Guía para las universidades, los centros de educación superior y el sector académico. Melbourne: SDSN.

UIL-UNESCO (2017). Ciudades del aprendizaje y los ODS: una Guía de Acción. Hamburgo: Instituto de la Unesco para el Aprendizaje a lo largo de toda la vida.

UNECE (2012). Learning for the future. Competences in Education for Sustanaible Development. Ginebra: Naciones Unidas.

UNECE (2013). Empowering educators for a sustainable future. Tools for policy and practice workshops on competences in education for sustainable development. Ginebra: Naciones Unidas.

UNESCO (2014). Hoja de ruta para la ejecución del programa de acción mundial de Educación para el Desarrollo Sostenible. Paris: UNESCO.

UNESCO (2015). Global citizenship education: Topics and learning objectives. Paris: UNESCO.

UNU-IAS (2014). Transforming Higher Education and Creating Sustainable Societies. Tokyo: UNU. 
SDSN (2017). Sustainable Development Solutions Network 